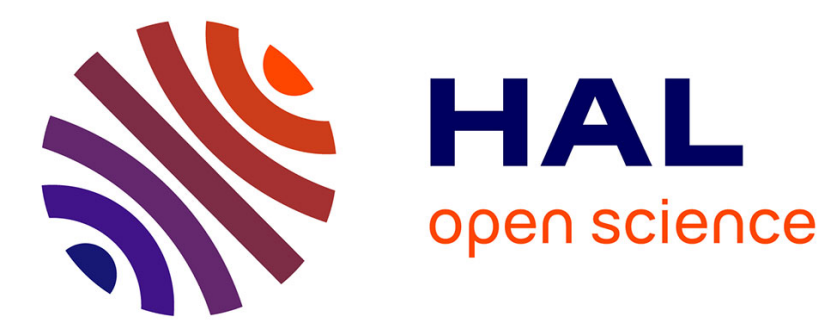

\title{
Linear-scaling total-energy calculations with the tight-binding Korringa-Kohn-Rostoker Green function method
}

Rudolf Zeller

\section{- To cite this version:}

Rudolf Zeller. Linear-scaling total-energy calculations with the tight-binding Korringa-KohnRostoker Green function method. Philosophical Magazine, 2008, 88 (18-20), pp.2807-2815. 10.1080/14786430802406256 . hal-00513960

\section{HAL Id: hal-00513960 \\ https://hal.science/hal-00513960}

Submitted on 1 Sep 2010

HAL is a multi-disciplinary open access archive for the deposit and dissemination of scientific research documents, whether they are published or not. The documents may come from teaching and research institutions in France or abroad, or from public or private research centers.
L'archive ouverte pluridisciplinaire HAL, est destinée au dépôt et à la diffusion de documents scientifiques de niveau recherche, publiés ou non, émanant des établissements d'enseignement et de recherche français ou étrangers, des laboratoires publics ou privés. 


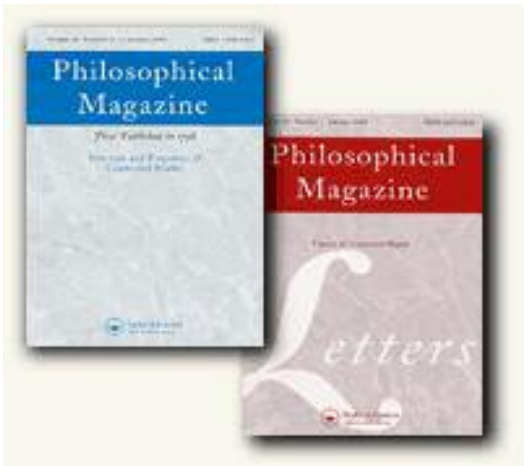

\section{Linear-scaling total-energy calculations with the tight-binding Korringa- Kohn-Rostoker Green function method}

\begin{tabular}{|r|l|}
\hline Journal: & Philosophical Magazine \& Philosophical Magazine Letters \\
\hline Manuscript ID: & TPHM-08-Jul-0250 \\
\hline Journal Selection: & Philosophical Magazine \\
\hline Author: & 10 -Jul-2008 \\
\hline Complete List of Authors: & $\begin{array}{l}\text { Zeller, Rudolf; Forschungszentrum Jülich GmbH, Institut für } \\
\text { Kestkörperforschung }\end{array}$ \\
\hline Keywords (user supplied): & electronic structure, metallic systems \\
\hline complex energies, linear scaling, iterative solution \\
\hline $\begin{array}{l}\text { Note: The following files were submitted by the author for peer review, but cannot be converted } \\
\text { to PDF. You must view these files (e.g. movies) online. }\end{array}$ \\
\hline zeller.tex
\end{tabular}

\section{↔ ScholarONE" \\ Manuscript Central}




\title{
RESEARCH ARTICLE
}

\section{Linear-scaling total-energy calculations with the tight-binding Korringa-Kohn-Rostoker Green function method}

\author{
Rudolf Zeller \\ Institut für Festkörperforschung, Forschungszentrum Jülich GmbH, D-52425 Jülich, \\ Germany \\ (Received 00 Month 200x; final version received 00 Month 200x)
}

\begin{abstract}
A complex-energy broadening scheme with quartic dependence on the broadening parameter is presented for Green-function density-functional electronic-structure calculations. The scheme is applied in a recently developed linear-scaling algorithm based on the tight-binding KorringaKohn-Rostoker Green function method where it leads to considerable computational saving compared to the standard Fermi-Dirac broadening with quadratic dependence. The linearscaling algorithm is applied for total-energy and spin-moment calculations for large supercells and the advantages of quartic broadening scheme are discussed.
\end{abstract}

Keywords: electronic structure; linear scaling; iterative solution; complex energies

\section{Introduction}

Standard density-functional calculations are usually restricted to about a thousand atoms even on the best supercomputers available today, since the computational effort increases with the third power of the number of atoms in the system. In the last years a number of codes have appeared [1-4] which overcome this bottleneck and achieve linear-scaling effort by utilizing the decay of the density matrix [5], which is exponential in semiconducting and insulating materials. For metallic systems, where (at zero temperature) the decay is only algebraical, a linear-scaling algorithm based on the tight-binding (TB) Korringa-Kohn-Rostoker (KKR) Green function method has been suggested recently [6]. This algorithm exploits the sparsity of the TB-KKR matrix, uses an iterative solution technique for the KKR matrix equations and applies a spatial truncation of the Green function to achieve linear scaling. For large $\mathrm{Cu}$ and $\mathrm{Pd}$ supercells as model systems it has been found [6] that the quasi-minimal residual (QMR) method [7, 8] leads to convergent iterations, if the charge density is obtained by complex-energy integration [9] with non-zero electronic temperature [10], and that the total-energy error introduced by the spatial truncation of the Green function is in the meV range, if the truncation region contains about a few thousand atoms.

Here the previous study, which was limited to the minimal choice $l_{\max }=2$ for the angular-momentum cut-off, is extended to $l_{\max }=3$, which is more reasonable for total-energy calculations, and to a spin-polarized material to illustrate that the algorithm is also useful for the calculation of spin moments. Since the main computational effort for the iterative solution appears at the complex energy with smallest imaginary part, which is proportional to temperature $T$, it is important that $T$ can be chosen as large as acceptable for the desired total-energy accuracy.

ISSN: 1478-6435 print/ISSN 1478-6443 online

(C) 200x Taylor \& Francis

DOI: $10.1080 / 1478643 \mathrm{YYxxxxxxxx}$

http://www.informaworld.com 
Therefore, it is one aim of this paper to present and explain a complex-energy

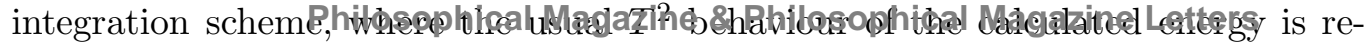
placed by a $T^{4}$ behaviour, and to show that this scheme considerably reduces the computational cost for the iterative solution.

\section{Basic equations}

In the full-potential KKR Green function method space is divided into nonoverlapping space-filling cells around the atomic positions $\boldsymbol{R}^{n}$. In each cell the Green function can be written [11] in a multiple-scattering representation as

$$
G\left(\boldsymbol{r}+\boldsymbol{R}^{n}, \boldsymbol{r}^{\prime}+\boldsymbol{R}^{n^{\prime}} ; E\right)=\delta^{n n^{\prime}} G_{s}^{n}\left(\boldsymbol{r}, \boldsymbol{r}^{\prime} ; E\right)+\sum_{L L^{\prime}} R_{L}^{n}(\boldsymbol{r} ; E) G_{L L^{\prime}}^{n n^{\prime}}(E) R_{L^{\prime}}^{n^{\prime}}\left(\boldsymbol{r}^{\prime} ; E\right)
$$

where $\boldsymbol{r}$ and $\boldsymbol{r}^{\prime}$ are cell centred coordinates and $L$ stands for the angular-momentum indices $l$ and $m$ and $\boldsymbol{r}$ and $\boldsymbol{r}^{\prime}$ are cell centred coordinates. $G_{s}^{n}\left(\boldsymbol{r}, \boldsymbol{r}^{\prime} ; E\right)$ and $R_{L}^{n}(\boldsymbol{r} ; E)$ denote single-scattering Green function and wavefunctions which depend only on the potential $V(\boldsymbol{r})$ inside cell $n$ and can be calculated with linear-scaling effort by solving single-site integral equations as described in [11, 12] or in [13]. For spinpolarized potentials the Green function and wavefunctions carry an additional spin index which, however, will be suppressed in the following to simplify the notation. The density $n\left(\boldsymbol{r}+\boldsymbol{R}^{n}\right)$ inside cell $n$ is obtained $[10,11]$ by integration

$$
n\left(\boldsymbol{r}+\boldsymbol{R}^{n}\right)=-\frac{1}{\pi} \operatorname{Im} \int_{-\infty}^{\infty} \mathrm{d} E f\left(E-E_{F}, T\right) G\left(\boldsymbol{r}+\boldsymbol{R}^{n}, \boldsymbol{r}+\boldsymbol{R}^{n} ; E+\mathrm{i} \epsilon\right),
$$

where $f\left(E-E_{F}, T\right)=\left(1+\exp \left(\left(E-E_{F}\right) / k T\right)\right)^{-1}$ is the Fermi-Dirac function for temperature $T$ and $E_{F}$ denotes the Fermi energy. The infinitesimal quantity $\epsilon \rightarrow 0+$ guarantees that the singularities of the Green function on the real energy axis are avoided and that the Green function in the correct energy half plane is used. The Green function matrix elements $G_{L L^{\prime}}^{n n^{\prime}}(E)$ are obtained by solving the matrix equation

$$
G_{L L^{\prime}}^{n n^{\prime}}(E)=G_{L L^{\prime}}^{r, n n^{\prime}}(E)+\sum_{n^{\prime \prime} L^{\prime \prime} L^{\prime \prime \prime}} G_{L L^{\prime \prime}}^{r, n n^{\prime \prime}}(E) \Delta t_{L^{\prime \prime} L^{\prime \prime \prime}}^{n^{\prime \prime}}(E) G_{L^{\prime \prime \prime} L^{\prime}}^{n^{\prime \prime} n^{\prime}}(E)
$$

where $G_{L L^{\prime}}^{r, n n^{\prime \prime}}(E)$ are the Green function matrix elements of suitably chosen reference system and $\Delta t_{L L^{\prime}}^{n}(E)$ are $t$-matrix differences which can be calculated from the potential and the reference potential, both restricted to cell $n$. The system (3) of linear equations represents an algebraic Dyson equation with complex, nonHermitian matrices of dimension $N\left(l_{\max }+1\right)^{2}$.

The standard reference system in the KKR method is free space. Here the Green function matrix elements $G_{L L^{\prime}}^{0, n n^{\prime}}(E)$, traditionally called structure constants, are known analytically, but decay unfavourably slowly with distance between site $n$ and $n^{\prime}$. Because of the slow decay all elements in the KKR matrix $t^{-1}-G^{0}$ have to be taken into account and because of that iterative solution of (3) is much more expensive than direct solution since both require a computational effort proportional to $N^{3}$, which must be repeated in each iteration, whereas it is needed only once for direct solution. A useful reference system [14] with rapidly decaying structure constants in the energy range relevant for density-functional calculations consists of an infinite array of repulsive potentials (here chosen with a constant height of 8 
Ry), which are confined to nonoverlapping muffin-tin spheres around the sites $\boldsymbol{R}^{n}$.

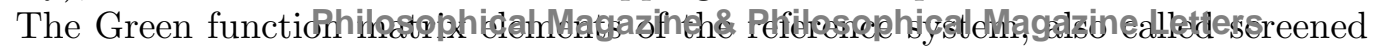
structure constants, can be calculated in real space with linear scaling effort by solving the Dyson equation

$$
G_{L L^{\prime}}^{r, n n^{\prime}}(E)=G_{L L^{\prime}}^{0, n n^{\prime}}(E)+\sum_{n^{\prime \prime} L^{\prime \prime} L^{\prime \prime \prime}} G_{L L^{\prime \prime}}^{0, n n^{\prime \prime}}(E) t_{L^{\prime \prime} L^{\prime \prime \prime}}^{r, n^{\prime \prime}}(E) G_{L^{\prime \prime \prime} L^{\prime}}^{r, n^{\prime \prime} n^{\prime}}(E)
$$

separately for each site $n^{\prime}$ by use of a finite cluster of $N_{c l}$ repulsive potentials with the $t$ matrices $t_{L^{\prime \prime} L^{\prime \prime \prime}}^{r, n^{\prime \prime}}(E)$. For the present study the choice $N_{c l}=13$ was used which includes the central site and its nearest neighbours. Due to the rapid decay of $G_{L^{\prime \prime \prime} L^{\prime}}^{r, n^{\prime \prime}}(E)$ with distance $\left|\boldsymbol{R}^{n^{\prime \prime}}-\boldsymbol{R}^{n^{\prime}}\right|$ other sites $n^{\prime \prime}$ were neglected in (4). This neglect reduces the dimension of the linear system (4) to $N_{c l}\left(l_{\max }+1\right)^{2}$, leads to a sparse TB-KKR matrix with sparsity degree $N_{c l} / N$ and makes the computational effort proportional to $N_{i t} N_{c l} N^{2}$ for the iterative solution of (3) compared to $N^{3}$ for the direct solution so that essentially $N^{2}$ scaling is achieved by iterative solution as has been discussed already by Smirnov and Johnson [15].

An important feature of iterative solutions is that for each site $n$ the Green function matrix elements can be determined independently so that iterative solutions are ideally suited for massively parallel computing. A straightforward iteration of (3) corresponds to the Born iteration of scattering theory which often diverges, in particular for energies with small imaginary part. This problem can be avoided if one of the known iterative solution techniques for systems of linear equations is used which works for non-Hermitian matrices. One such technique, which was used successfully in the current investigation, is the quasi-minimal residual (QMR) method $[7,8]$ in its transpose free version, which was used also by Smirnov and Johnson [15].

In order to arrive at a linear-scaling algorithm the principle of nearsightedness of electronic matter $[16,17]$, which is exploited in other linear-scaling methods $[1-5]$ for the density matrix, was used for the Green function $G\left(\boldsymbol{r}, \boldsymbol{r}^{\prime} ; E\right)$ which decays at the complex energy mesh points in a similar fashion as the finite-temperature single-particle density matrix $\rho\left(\boldsymbol{r}, \boldsymbol{r}^{\prime}, T\right)$ as can be seen from the equation

$$
\rho\left(\boldsymbol{r}, \boldsymbol{r}^{\prime}, T\right)=-\frac{1}{\pi} \operatorname{Im} \int_{-\infty}^{\infty} \mathrm{d} E f\left(E-E_{F}, T\right) G\left(\boldsymbol{r}, \boldsymbol{r}^{\prime} ; E\right) .
$$

If this integral is evaluated by using the integrand at the Matsubara energies and on the complex-energy contour as described in the appendix, the decay of $\rho\left(\boldsymbol{r}, \boldsymbol{r}^{\prime}, T\right)$ is mainly determined by the decay of $G\left(\boldsymbol{r}, \boldsymbol{r}^{\prime} ; E_{F}+\mathrm{i} \pi k T\right)$, where $E_{F}+\mathrm{i} \pi k T$ is the first Matsubara energy with smallest imaginary part, since the Green function decays faster on all other integration mesh points $E_{i}$ which have larger values of $\operatorname{Im} E_{i}$. Thus a neglect of the Green function in (2) for large distances $\left|\boldsymbol{r}-\boldsymbol{r}^{\prime}\right|$ corresponds to a neglect of the finite-temperature density matrix for similar distances.

Since the single-scattering wavefunctions in (1) are only multiplicative factors, a truncation of the Green function directly corresponds to a neglect of Green function matrix elements $G_{L L^{\prime}}^{n n^{\prime}}$ beyond a chosen distance $d_{c u t}$, which means that in (3) only $O\left(N_{t r} N\right)$ elements $G_{L L^{\prime}}^{n n^{\prime}}$ appear instead of $O\left(N^{2}\right)$ so that the computational effort is reduced by a factor $N_{t r} / N$. Here $N_{t r}$ is the number of atoms which are included in the truncation region given by $\left|\boldsymbol{R}^{n}-\boldsymbol{R}^{n^{\prime}}\right|<d_{\text {cut }}$. The total effort for all $N$ atoms is then proportional to $N_{i t} N_{c l} N_{t r} N$. This increases linearly with $N$ since $N_{i t}$ approaches a constant value for large $N$ as will be illustrated in the next section. It should be pointed out that because of the singularities of the Green function 


\begin{tabular}{lcccccc}
\hline & $N_{i t}^{\infty}(800)$ & $\alpha(800)$ & $\gamma(800)$ & $N_{i t}^{\infty}(1600)$ & $\alpha(1600)$ & $\gamma(1600)$ \\
\hline $\mathrm{Ni}^{-}$ & 7568 & 7679 & -0.0066 & 694 & 781 & -0.0506 \\
$\mathrm{Ni}^{+}$ & 1151 & 1292 & -0.0401 & 518 & 597 & -0.0591 \\
$\mathrm{Cu}$ & 1347 & 1630 & -0.0537 & 519 & 707 & -0.0985 \\
$\mathrm{Pd}$ & 2339 & 2563 & -0.0271 & 566 & 673 & -0.0661 \\
\hline
\end{tabular}

on the real energy axis the use of complex energies is unavoidable and that the required number $N_{i t}$ of iterations increases with decreasing imaginary part of $E_{i}$.

\section{Total-energy results and iteration behaviour}

Previously [6] the proposed linear-scaling algorithm has been studied for large $\mathrm{Cu}$ and $\mathrm{Pd}$ supercells for the minimal choice $l_{\max }=2$ of the angular-momentum cut-off. Here similarly large supercells were studied with $l_{\max }=3$, which is more relevant for total-energy calculations. The supercells contained $4 \times 32^{3}=131072$ atoms arranged in the appropriate face-centred-cubic geometry and were obtained by repeating a simple cubic unit with four atoms 32 times in the three space directions. A single point $\boldsymbol{k}=(1 / 4,1 / 4,1 / 4) \times 2 \pi / a$ was used in the irreducible part of the Brillouin zone. The supercell lattice constant $a$ was chosen as 11.276, 11.568 and $124.74 \mathrm{~nm}$ for $\mathrm{Ni}, \mathrm{Cu}$ and $\mathrm{Pd}$, which is 32 times the experimental lattice constant of these elements. Since all atoms in the supercells are equivalent, the iterative solution of the Dyson equation (3) is needed for only one value of $n^{\prime}$. This represents an enormous reduction of the computational effort in the present model study compared to realistic systems with inequivalent atoms. According to the concept of equivalent $\boldsymbol{k}$ point meshes [18] the self-consistent potentials for the supercells agree with the ones of the simple cubic unit cells with four atoms if 5984 $\boldsymbol{k}$ points in the irreducible part of the simple cubic Brillouin zone are used. This means that self-consistent potentials for the large supercells could be obtained in an rather inexpensive way.

An important issue for the proposed algorithm is how fast the iterations converge. The main computational work consists in matrix-vector multiplications, which were repeated for each site $n^{\prime}$ and angular-momentum component $L^{\prime}$ independently until the solution is obtained with the prescribed precision, here specified by the residual norm $\|r\|=10^{-6}$ for the QMR iterations. The number $N_{i t}$ of iterations depends on system size and on the imaginary part of the energy $E_{i}$. The highest number of iterations is required at the first Matsubara energy $E_{F}+\mathrm{i} \pi k T$. In the previous investigation [6] for $l_{\max }=2$ it has been found that the dependence of $N_{i t}$ on the number $N_{t r}$ of atoms in the truncation region could be fitted to an exponential function of the form

$$
N_{i t}\left(N_{t r}\right)=N_{i t}^{\infty}-\alpha \exp \left(\gamma N_{t r}^{1 / 3}\right)
$$

with three temperature dependent parameters $N_{i t}^{\infty}, \alpha$ and $\gamma$. In the present study for $l_{\max }=3$ the same behaviour was found for $T=800 \mathrm{~K}$ and $T=1600 \mathrm{~K}$. For truncation regions with more than 20000 atoms, the results of $N_{i t}\left(N_{t r}\right)$ for $T=400 \mathrm{~K}$ scattered too much so that the fits became ill-defined and reliable fit parameters could not be extracted. Note that the values for $N_{i t}^{\infty}$ given in Table 1 , which correspond to averages over the $\left(l_{\max }+1\right)^{2}=16$ angular-momentum components, are larger than the values obtained previously for $l_{\max }=2$. However, 


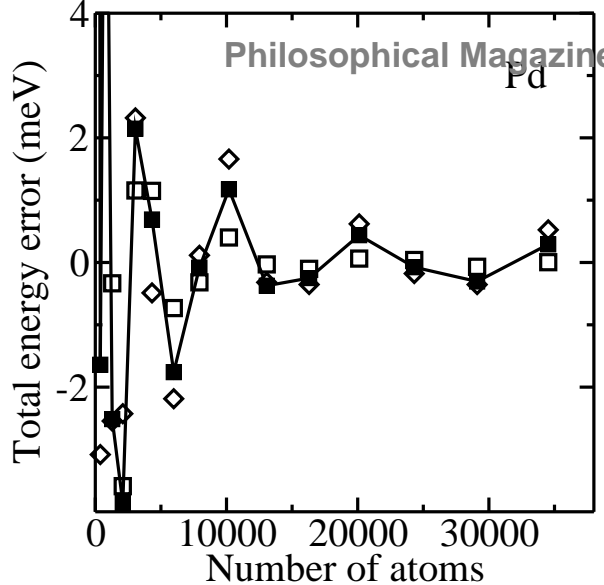

(a)

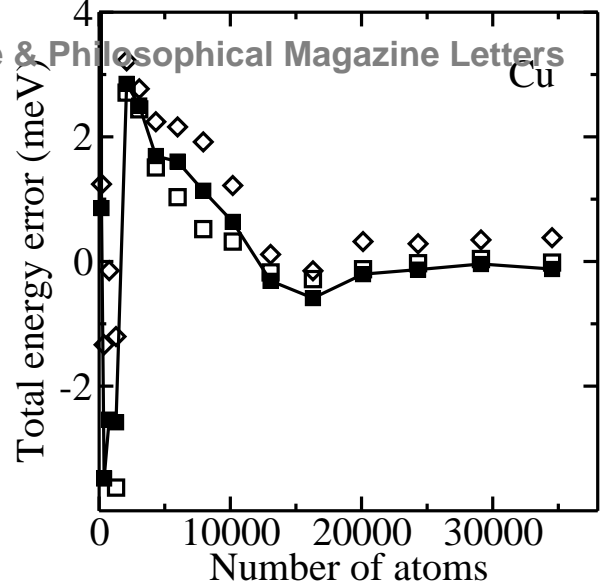

(b)

Figure 1. Total-energy error per atom as function of the number of atoms contained in the truncation region. Solid and open squares are for $T=800 \mathrm{~K}$ and $1600 \mathrm{~K}$, diamonds for $T=400 \mathrm{~K}$. The lines, which connect the results for $T=800 \mathrm{~K}$, serve as guide for the eye.

those values, which stood for the number of QMR iterations, cannot be compared directly to the ones in Table 1 , since here the more meaningful numbers of matrixvector multiplications are displayed which are about twice as large as the numbers of calls to the QMR subroutines displayed previously.

An important issue for the proposed linear-scaling algorithm concerns the question how accurate the total energy can be calculated if spatial truncation for the Green function is applied. Figure 1 shows the total-energy error as function of the number $N_{t r}$ of atoms in the truncation region and illustrates that the error is smaller than $2 \mathrm{meV}$ if truncation regions with a few thousand atoms are used and that the proposed algorithm thus can be expected to be useful for linearscaling total-energy calculations for large metallic systems. Another effect seen in figure 1 is that, for truncation regions with up to 10000 atoms, higher electronic temperature does not lead to substantially lower total-energy errors. Probably the zero-temperature algebraical decay of the Green function (and density matrix) dominates the additional exponential decay caused by temperature up to truncation distances of approximately 10 times the face-centred-cubic lattice constant.

It should be mentioned that it was not necessary to make self-consistent calculation for the total-energy error caused by the truncation because the modified energy functional $\tilde{E}[n(\boldsymbol{r})]=E[n(\boldsymbol{r})]-E_{F}\left(\int n(\boldsymbol{r})-N_{e l}\right)$ was used instead of the standard energy functional $E[n(\boldsymbol{r})]$. This functional is extremal for density variations, even if the total number $N_{e l}$ of electrons changes, and has been demonstrated to yield accurate results in impurity calculations [19], where charge-neutrality deviations of about 0.01 to 0.1 electrons exist.

\section{Quartic broadening scheme}

From the results in Table 1 it is clear that the computational effort, which is proportional to the number of iterations (matrix-vector multiplications), is considerably reduced for higher electronic temperature. It has been argued [20] that large temperature can be detrimental for total-energy calculations, in particular for magnetic systems, where magnetism vanishes at higher temperature. Therefore, if one is interested in the total energy $E_{t o t}=E(0)$, it is advantageous to replace the 
the truncation region contains about a few thousand atoms. It would be desirable

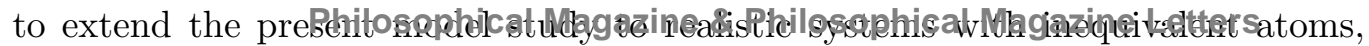
where obviously much more computing power is needed, which is expected to be available on massively parallel supercomputers.

\section{References}

\section{References}

[1] J. M. Soler, E. Artacho, J. D. Gale, A. Garcia, J. Junquera, P. Ordejon, and D. Sanchez-Portal, J. Phys.: Condens. Matter, 14, 2745 (2002).

[2] D. R. Bowler, T. Miyazaki, and M. J. Gillan, J. Phys.: Condens. Matter, 14, 2781 (2002).

[3] C-K. Skylaris, P. D. Haynes, A. A. Mostofi, and M. C. Payne, J. Chem. Phys., 122, 084119 (2005).

[4] T. Ozaki and H. Kino, Phys. Rev. B, 72, 045121 (1995).

[5] S. Goedecker, Rev. Mod. Phys., 71, 1085 (1999).

[6] R. Zeller, J. Phys.: Condens. Matter, 20, 294215 (2008).

[7] R. W. Freund and N. M. Nachtigal, Numer. Math., 60, 315 (1991).

[8] R. W. Freund, SIAM J. Sci. Comput., 14, 470 (1993).

[9] R. Zeller, J. Deutz, and P. H. Dederichs, Solid State Commun., 44, 993 (1982).

[10] K. Wildberger, P. Lang, R. Zeller, and P. H. Dederichs, Phys. Rev. B, 52, 11502 (1995).

[11] N. Papanikolaou, R. Zeller, and P. H. Dederichs, J. Phys.: Condens. Matter, 14, 2799 (2002).

[12] B. Drittler, M. Weinert, R. Zeller, and P.H. Dederichs, Solid State Commun., 79, 31 (1991).

[13] J. Zabloudil, R. Hammerling, L. Szunyogh, and P. Weinberger, Electron Scattering in Solid Matter: A Theoretical and Computational Treatise, Springer Series in Solid-State Sciences , Vol. 147, Springer, Berlin Heidelberg New York, 2005.

[14] R. Zeller, P. H. Dederichs, B. Újfalussy, L. Szunyogh, and P. Weinberger, Phys. Rev. B, 52, 8807 (1995).

[15] A. V. Smirnov and D. D. Johnson, Comput. Phys. Commun., 148, 74 (2002).

[16] E. Prodan E and W. Kohn, Proc. Natl. Acad. Sci. USA, 102, 11635 (2005).

[17] W. Kohn, Phys. Rev. Lett., 76, 3168 (1996).

[18] N. Chetty, M. Weinert, T. S. Rahman, and J. W. Davenport, Phys. Rev. B, 52, 6313 (1989).

[19] B. Drittler, M. Weinert, R. Zeller, and P. H. Dederichs, Phys. Rev. B, 39, 930 (1989).

[20] A. V. Smirnov and D. D. Johnson, Phys. Rev. B, 64, 235129 (2002).

[21] N. W. Ashcroft and N. D. Mermin, Solid State Physics: Advances in Research and Applications, Holt, Rinehart and Winston, New York, 1976, p. 45.

[22] M. Methfessel and A. T. Paxton, Phys. Rev. B, 40, 3616 (1989).

[23] N. Marzari, D. Vanderbilt, A. de Vita, and M. C. Payne, Phys. Rev. Lett., 82, 3296 (1999).

[24] M. J. Gillan, J. Phys.: Condens. Matter, 1, 689 (1989).

[25] W. Gautschi, Orthogonal Polynomials: Computation and Approximation, Oxford University Press, Oxford New York, 2004.

\section{Appendix A. Construction of complex-energy integration rules}

Instead of integrating (2) along the real energy axis, the integral can be evaluated in the complex energy plane because the Green function is an analytical function except for singularities for real energies. For the present study the contour starts on the negative real energy axis at energy $E_{0}$ below the valence and above the core states and thus includes only contributions of valence states. Contributions of core states were calculated separately by treating these states in an atomic like fashion. From $E_{0}$ the contour goes parallel to the imaginary axis up to $E_{0}+2 J \mathrm{i} \pi k T$, where $J$ was a chosen small even integer. On this part of the contour the Fermi-Dirac function was replaced by the constant value 1 since for the temperatures used the quantity $\exp \left(\left(E_{0}-E_{F}\right) /(2 k T)\right)$ was smaller than $10^{-20}$. From $E_{0}+2 J \mathrm{i} \pi k T$ the contour goes parallel to the real axis to infinity. Compared to the integral from $E_{0}$ to infinity along the real axis, the integral on the contour differs by the contribution of the residues arising from the poles of the Fermi-Dirac function at the first $J$ Matsubara energies $E_{j}=E_{F}+(2 j-1) \mathrm{i} \pi k T$ with $j=1,2, \ldots, J$, which are added separately. Compared to other broadening functions [22, 23] the Fermi-Dirac function is more advantageous in the complex-energy plane despite the necessity to include pole contributions, which do not exist if a Gauss function is used. Due to 
the exponential dependence on $E$, both functions have high amplitude oscillations

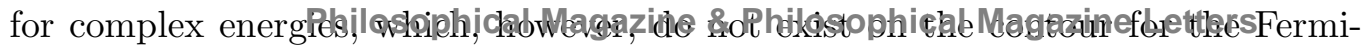
Dirac function because of its periodicity $f(E+2 J \mathrm{i} \pi k T, T)=f(E, T)$ so that the Fermi-Dirac function on the contour is real and as well behaving as on the real energy axis.

For the numerical integration the contour interval from $E_{0}+2 J i \pi k T$ to infinity was divided into several subintervals. On part of these subintervals the Fermi-Dirac function could be replaced by 1 and standard Gauss-Legendre integration rules could be used, whereas for other subintervals special Gauss rules had to be constructed for the weight functions $w_{1}(E)=f\left(E-E_{F}, T\right)$ and $w_{2}(E)=4 f\left(E-E_{F}, T\right) / 3-f\left(E_{F}-E, 2 T\right) / 3$. For $w_{1}(E)$ one subinterval was chosen from $E_{F}+2 J i \pi k T$ to infinity and for $w_{2}(E)$ from $E_{F}+x_{0}+2 J i \pi k T$ to infinity. Here $x_{0}=2 \ln (2+\sqrt{7}) k T \approx 3.071906 k T$ is the only point on the contour, where $w_{2}(E)$ changes sign, so that the construction of Gauss rules was not complicated by sign changes of the weight function. Thus the correct choice of subintervals in the complex-energy plane with $x_{0}$ being at the boundary of two subintervals completely removes the problem of negative values of the broadening function which leads to the trouble of negative occupancies in basis-set methods [23]. The construction of Gauss integration rules is straightforward (see e. g. [25]), although numerical steps might be susceptible to rounding errors because of illconditioning. Here the rules were constructed according to the following prescription. Monic orthogonal polynomials $p_{n}(x)=x^{n}+\ldots$ satisfy a three term recurrence $p_{n+1}(x)=\left(x-\alpha_{n}\right) p_{n}(x)-\beta_{n} p_{n-1}(x)$ starting with $p_{-1}=0$ and $p_{0}=1$, where the coefficients are given by $\alpha_{n}=C_{n}^{-1} \int x p_{n}^{2}(x) w(x) \mathrm{d} x$ and $\beta_{n}=C_{n-1}^{-1} C_{n}$ with $C_{n}$ defined as $C_{n}=\int p_{n}^{2}(x) w(x) \mathrm{d} x$ and $\beta_{0}=\int w(x) \mathrm{d} x$. For an $\mathrm{N}$ point integration rule the mesh points $x_{i}$ are given by the roots of the polynomial $p_{N}(x)$ and the integration weights are determined by

$$
w_{i}=\frac{C_{n-1}}{p_{N}^{\prime}\left(x_{i}\right) p_{N-1}\left(x_{i}\right)},
$$

where the prime denotes derivative with respect to $x$. Numerically the values of $x_{i}$ and $w_{i}$ were obtained by a computer algebra system with 90 digits precision to avoid rounding error problems. In total the number of Gauss points on the contour was about 50, which kept the total-energy errors arising from energy integration below $1 \mu \mathrm{Ry}$. 


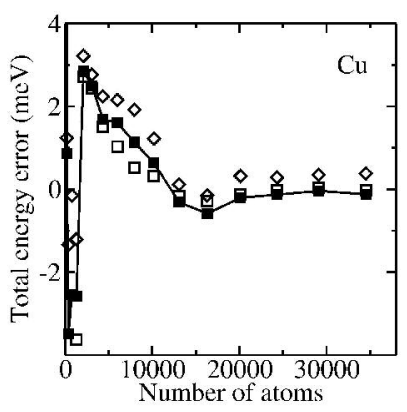

$215 \times 279 m m(600 \times 600$ DPI $)$ 


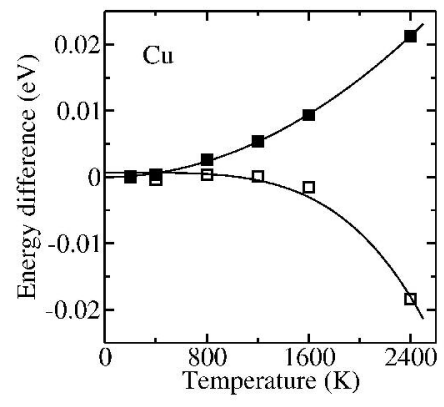

$215 \times 279 \mathrm{~mm}(600 \times 600 \mathrm{DPI})$ 


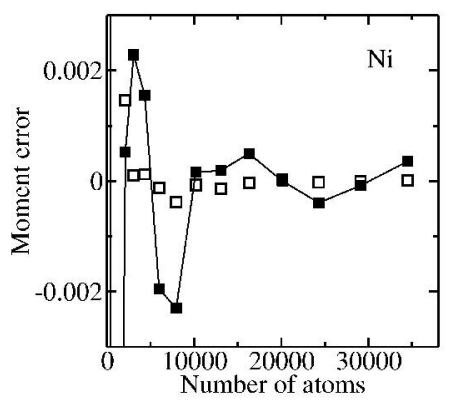

$215 \times 279 \mathrm{~mm}(600 \times 600 \mathrm{DPI})$ 


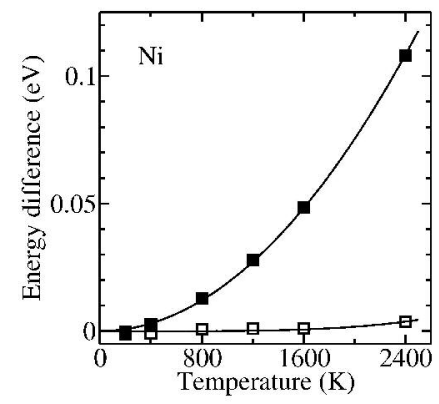

$215 \times 279 \mathrm{~mm}(600 \times 600 \mathrm{DPI})$ 


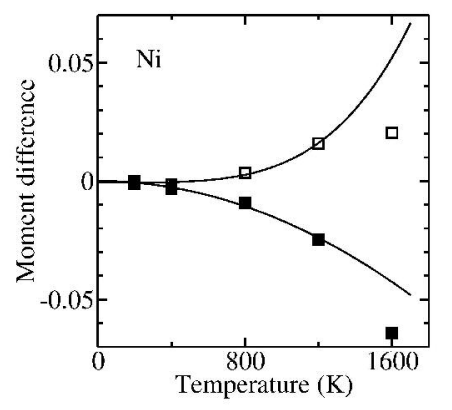

$215 \times 279 \mathrm{~mm}(600 \times 600 \mathrm{DPI})$ 


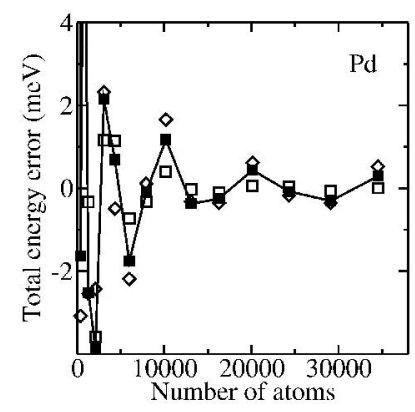

$215 \times 279 m m(600 \times 600$ DPI $)$ 